\title{
Confiabilidad y validez de la Escala de Estrategias de Aprendizaje ACRA en estudiantes universitarios del Estado de México
}

Carlos Saúl Juárez Lugo, ${ }^{1}$ Karina Pichardo Silva, ${ }^{1}$ María del Consuelo Escoto Ponce de León ${ }^{1}$

y Elba Luna Montijo ${ }^{2}$

\section{Introducción}

Para Monereo (2004), las estrategias de aprendizaje son un proceso de toma de decisiones, conscientes e intencionales, con las cuales el alumno elige y recupera, de manera coordinada, los conocimientos conceptuales, procedimentales y actitudinales necesarios para cumplir un determinado objetivo, siempre en función de las condiciones de la situación educativa en que se produce la acción. La función primordial de las estrategias en todo proceso de aprendizaje es facilitar la asimilación de la información que llega del exterior al sistema cognitivo del alumno. Este proceso implica la gestión y supervisión de los datos que entran, así como la clasificación, la categorización, almacenamiento, recuperación y salida de la información.

De acuerdo con Coll (2001), Gargallo (2002) y Monereo (1990), en las últimas dos décadas, la investigación sobre las

1 Universidad Autónoma del Estado de México.

2 Sistema Educativo del Estado de México. 
estrategias de aprendizaje en el ámbito escolar ha tenido una atención especial como uno de los factores relevantes de la educación formal, por ejemplo, en el tratamiento curricular de los contenidos procedimentales, en la práctica del profesor como facilitador de aprendizaje y en el impacto que las estrategias pueden tener en el alumno para construir un tipo de aprendizaje más comprensivo, eficaz y óptimo.

Entre las pruebas existentes para medir este constructo, la Escala de Estrategias de Aprendizaje ACRA, elaborada por Román y Gallego (1994), es uno de los instrumentos más utilizados. La Escala ACRA originalmente fue diseñada para ser aplicada a niños de entre 12 a 16 años con el objetivo de identificar los procesos que intervienen en el aprendizaje y que dan nombre a las cuatro escalas que la conforman: adquisición, codificación, recuperación y apoyo.

En México, así como en otros países de habla hispana, se ha utilizado la Escala de Estrategias de Aprendizaje ACRA (Román y Gallego, 1994) para evaluar los procesos cognitivos en estudiantes universitarios, sin considerar que la escala fue diseñada para estudiantes españoles de educación secundaria obligatoria. En la mayoría de las investigaciones con universitarios se utiliza la versión original del ACRA para relacionar el uso de las estrategias con variables como el sexo, el semestre que cursa el alumno, la formación profesional y el rendimiento académico (Bernal, 2009; Camarero, Martín y Herrero, 2000; Cardozo et al., 2011; Cruz y Anzaldo, 2009; Juárez, Rodríguez y Luna, 2012; Martín y Camarero, 2001). En otras investigaciones con estudiantes universitarios se presentan versiones abreviadas de este instrumento, pero no reportan de manera puntual el procedimiento psicométrico con el que fue reducida la escala (De la Fuente y Justicia, 2003; Gutiérrez, 2009; Villamizar, 2008).

Ante este panorama, la presente investigación tiene por objetivo conocer las propiedades psicométricas de la Escala de Estrategias de Aprendizaje ACRA (Román y Gallego, 1994) en una muestra de estudiantes mexicanos a nivel universitario, debido a que no existen estudios reportados al respecto en nuestro país. 


\section{Método}

Participantes

La muestra estuvo compuesta por 623 estudiantes universitarios (67.1\% mujeres y $32.9 \%$ varones), con una edad comprendida en un rango de 17 a 44 años $(M=18.74$ años; $D E=2.53)$ de seis licenciaturas.

\section{Instrumentos}

La Escala de Estrategias de Aprendizaje ACRA que se utiliza en esta investigación fue elaborada por Román y Gallego (1994), fue construida para ser utilizada de forma preferente en estudiantes de enseñanza secundaria obligatoria (12 a 16 años aproximadamente). Evalúa el uso que habitualmente hacen los estudiantes de las estrategias de aprendizaje. Está conformada por cuatro escalas independientes, que son adquisición, codificación, recuperación de la información (procesos cognitivos) y apoyo al procesamiento (procesos metacognitivos y afectivos), 32 factores (estrategias de aprendizaje) y 119 ítems (técnicas de aprendizaje) con cuatro opciones de respuesta ( $A=$ nunca o casi nunca; $\mathrm{D}=$ siempre o casi siempre).

El Cuestionario Honey-Alonso de Estilos de Aprendizaje CHAEA (Alonso, Gallego y Honey, 2007), que evalúa los estilos de aprendizaje, está conformado por 80 reactivos, de los cuales 20 corresponden a cada uno de los estilos de aprendizaje, activo, reflexivo, teórico y pragmático, los cuales están distribuidos aleatoriamente, a las que se responde dicotómicamente, manifestando si se está de acuerdo (signo +) o en desacuerdo (signo -).

El cuestionario de Estrategias de Aprendizaje para universitarios CEA-U (Martin, García, Torbay y Rodríguez, 2007) consta de un total de 57 ítems que hacen referencia a diversas estrategias. Consta de tres escalas: estrategias motivacionales (27 ítems), estrategias cognitivas (22 ítems) y estrategias metacognitivas (8 ítems) con cinco alternativas de respuesta. 


\section{Resultados}

El coeficiente de confiabilidad total del ACRA es alto $(\alpha=.97)$, en lo que respecta a la consistencia interna de las cuatro escalas se obtuvieron puntajes altos: Adquisición $(\alpha=.80)$, Codificación $(\alpha=.93)$, Recuperación $(\alpha=.86)$ y Apoyo $(\alpha=.92)$.

Las puntuaciones del ACRA correlacionaron significativamente con la puntuación del CEA-U $(r=.470, p=.0001)$, lo cual indica que el ACRA presentó una adecuada validez concurrente.

Las puntuaciones del ACRA obtuvieron una correlación débil respecto a la puntuación del CHAEA $(r=.297, p=.0001)$, lo cual indica que el ACRA presentó una adecuada validez discriminante.

El Análisis Factorial (AF) se realizó para cada una de las escalas de forma independiente, se contemplaron tres criterios para llevar a cabo el AF: saturación del ítem $\geq .40$, un mínimo de tres ítems por factor y congruencia conceptual.

En la escala de estrategias de adquisición, el AF arrojó cuatro factores, los cuales explican $42.46 \%$ de la varianza. El AF agrupó a 15 ítems de los 20 ítems originales de esta escala. En la escala de codificación el AF arrojó siete factores que explican 53.90\% de la varianza total. De esta forma agrupó a 35 de los 46 ítems originales de esta escala. En cuanto a la escala de recuperación, el AF arrojó cuatro factores que explican $50.99 \%$ de la varianza. Agrupó a 17 de los 18 ítems originales de la escala original. Por último, la escala de apoyo al procesamiento arrojó una solución factorial compuesta por siete factores que explican $56.79 \%$ de la varianza. Se agrupó a 28 de los 35 reactivos originales de la escala.

\section{Conclusiones}

Con base en los resultados obtenidos, es posible concluir que la Escala de Estrategias de Aprendizaje ACRA tiene una alta consistencia interna para el instrumento en su totalidad, así como para sus escalas.

La estructura de la Escala de Estrategias de Aprendizaje ACRA, en su versión original, cuenta con 32 factores distribui- 
dos en cuatro escalas (Román y Gallego, 1994). Los resultados obtenidos del análisis factorial de componentes principales con rotación varimax de la presente investigación arrojaron una solución de 21 factores; estos valores sugieren que la diferencia de estructura factorial podría deberse a que el instrumento fue diseñado originalmente para alumnos de educación secundaria; sin embargo, se ha utilizado en varias investigaciones con estudiantes universitarios sin que se haya comprobado la pertinencia psicométrica del instrumento.

Coincidimos con De la Fuente y Justicia (2003) al señalar la inadecuación de la estructura factorial general del instrumento original para ordenar las técnicas utilizadas por estudiantes universitarios. El análisis factorial efectuado en este trabajo sugiere que el sistema educativo español y mexicano difiere en la implementación didáctica que hacen los profesores de los saberes que tiene que aprender el alumno. Mientras que en el ACRA las estrategias de aprendizaje distinguen distintas técnicas específicas, en el contexto mexicano las técnicas se agrupan de forma simplificada. Por ejemplo, el subrayado en el contexto español se divide en lineal e idiosincrático, mientras que en el contexto mexicano agrupó las dos técnicas anteriores más el epigrafiado en un solo factor. El universitario mexicano, de acuerdo a los resultados, no discrimina necesariamente las diferentes técnicas que se atribuyen a una estrategia, debido posiblemente a que su estructura cognitiva es más experta y pragmática al ejecutar procesos específicos de aprendizaje.

La validez concurrente del ACRA fue analizada mediante la correlación de la puntuación en esta prueba con la puntuación del CEA-U. Se encontró que la correlación fue estadísticamente significativa, lo cual indica que los procesos cognitivos de adquisición, codificación, recuperación de la información y apoyo al procesamiento se asocian con las estrategias motivacionales, cognitivas y metacognitivas de aprendizaje de los universitarios.

En cuanto a la validez discriminante del ACRA con la puntuación del CHAEA se encontró una correlación pobre entre ambas pruebas, lo cual nos indicaría que el ACRA discrimina entre el constructo de estrategias de aprendizaje y estilo de aprendizaje. 
En conclusión, el ACRA parece ser un instrumento adecuado para medir las estrategias de aprendizaje en estudiantes universitarios; sin embargo, consideramos necesario realizar una adaptación de la Escala de Estrategias de Aprendizaje ACRA a población universitaria mexicana, de acuerdo con los resultados de las propiedades psicométricas de la presente investigación. Es necesario realizar una prueba simplificada y contextualizada de los 119 ítems y 32 factores atendiendo los rasgos particulares del nivel educativo y del proceso enseñanza aprendizaje del país, así como explorar si dichos resultados se presentan también en educación secundaria y preparatoria.

\section{Referencias}

Alonso, C.M., Gallego, D.G. y Honey, P. (2007). Los estilos de aprendizaje. Procedimientos de diagnóstico y mejora. España: Ediciones Mensajero.

Bernal, F. (2009). Estrategias de aprendizaje en estudiantes de carreras pedagógicas y no pedagógicas de la universidad de Playa Ancha de CS. De la Educación. Revista de Orientación Educacional, 43, 35-57. Recuperado de http://dialnet.unirioja.es/servlet/articulo?codigo $=3098407$.

Camarero, F., Martín, F. y Herrero, J. (2000). Estilos y estrategias de aprendizaje en estudiantes universitarios. Psicothema, 12(4), 615-622.

Cardozo, D., Pérez, M.A., Jaramillo, M., Mendoza, R., Santillán, G. y Bobadilla, S. (2011). Estrategias de aprendizaje y rendimiento académico en estudiantes de la licenciatura en Psicología del Cu UAEM Temascaltepec. Revista de Educación y Desarrollo, 18, 35-40.

Coll, C. (2001). Psicología y currículum. Una aproximación psicopedagógica a la elaboración del currículum escolar. Barcelona: Laia.

Cruz, X. y Anzaldo, R. I. (septiembre, 2009). ¿Qué estrategias de aprendizaje utilizan los estudiantes de la LIE? El caso de la UPN unidad 142. Trabajo presentado el X Congreso Nacional 
de Investigación Educativa, área 1: aprendizaje y desarroIlo humano, Veracruz, Ver. Resumen recuperado de http:// www.comie.org.mx/congreso/memoriaelectronica/v10/contenido/contenido0101T.htm.

De la Fuente, J. y Justicia, F. (2003). Escala de estrategias de aprendizaje. ACRA-Abreviada para alumnos universitarios. Revista Electrónica de Investigación Psicoeducativa y Psicopedagógica, 1, 139-158.

Gargallo, B. (2002). Procedimientos. Estrategias de aprendizaje. Su naturaleza, enseñanza y evaluación. España: Humanidades Pedagogía.

Gargallo, B., Suárez, J. y Pérez, C. (2009). El cuestionario CEVEAPEAU. Un instrumento para la evaluación de las estrategias de aprendizaje de los estudiantes universitarios. Revista Electrónica de Investigación y Evaluación Educativa, 15(2), 1-31. Recuperado de http://www.uv.es/RELIEVE/v15n2/RELIEVEv15n2 5.htm.

Gutiérrez, R. (septiembre, 2009). Estrategias de aprendizaje en alumnos de una licenciatura en Psicología. Un estudio exploratorio. Trabajo presentado en el $X$ Congreso Nacional de Investigación Educativa, área 1: aprendizaje y desarroIlo humano, Veracruz, Ver. Resumen recuperado de http:// www.comie.org.mx/congreso/memoriaelectronica/v10/ contenido/contenido0101T.htm.

Juárez, C., Rodríguez, G. y Luna, L. (2012). El cuestionario de estilos de aprendizaje CHAEA y la Escala de Estrategias de Aprendizaje ACRA como herramienta potencial para la tutoría académica. Revista Estilos de Aprendizaje, 10(10), 1-31. Recuperado de http://www.estilosdeaprendizaje.es/.

Martín, E., García, L.A., Torbay, A. y Rodríguez, B. (2007). Estructura factorial y fiabilidad de un cuestionario de estrategias de aprendizaje en universitarios: CEA-U. Anales de Psicología, 23, 1-6.

Martín, F. y Camarero, F. (2001). Diferencias de género en los procesos de aprendizaje en universitarios. Psicothema, 13(4), 598-604. 
Monereo, C. (1990). Las estrategias de aprendizaje en la educación formal: enseñar a pensar y sobre el pensar. Infancia y Aprendizaje, 50, 3-25.

Monereo, C. (2004). Estrategias de aprendizaje y aprendizaje. Formación del profesorado y aplicación en la escuela $\left(10^{\mathrm{a}}\right.$ ed.). España: Graó.

Román, J. M. y Gallego, S. (1994). ACRA: Escala de Estrategias de Aprendizaje. Madrid: TEA Ediciones.

Villamizar, G. (2008). Relación entre estrategias de aprendizaje y rendimiento académico en estudiantes de Psicología. Docencia Universitaria, 9, 71-94. 